\title{
An Analysis of the Initiation Process of Electro-explosive Devices
}

\author{
Paulo Cesar de Carvalho Faria*, Koshun Iha, José Atílio Fritz Fidel Rocco \\ Instituto Tecnológico de Aeronáutica - São José dos Campos/SP - Brazil
}

Abstract: Electro-explosive devices (an electric resistance encapsulated by a primary explosive) fundamentally convert electrical energy into thermal energy, to start off an explosive chemical reaction. Obviously, the activation of those devices shall not happen by accident or, even worse, by intentional exogenous influence. From an ordinary differential equation, which describes the electro-explosive thermal behavior, a remarkable, but certainly not intuitive, dependence of the temperature response on the time constant of the heat transfer process is verified: the temperature profile dramatically changes as the time constant spans a wide range of values, from much lesser than the pulse width to much greater than the pulse period. Based on this dependence, important recommendations, concerning the efficient and safety operation of electro-explosive devices, are proposed.

Keywords: Electro-Explosive Devices, Squib, Thermal Model, Pulsed Initiation, Temperature Stacking.

\section{LIST OF SYMBOLS}

\begin{tabular}{|c|c|c|c|c|c|}
\hline$a=1 / \tau_{E E}$ & Inverse of the EED time constant & $\left(\mathrm{s}^{-1}\right)$ & $t$ & Time & $(\mathrm{s})$ \\
\hline ave & Average & $(--)$ & $T$ & Pulse period & $(\mathrm{s})$ \\
\hline$b$ & Barrel & $(--)$ & THRESHOLD & Ignition temperature threshold & $(---)$ \\
\hline$C_{T}$ & EED thermal capacitance & $\left(\mathrm{J} .{ }^{\circ} \mathrm{C}^{-1}\right)$ & $\wedge$ & And & $(---)$ \\
\hline$D C=P_{W} / T$ & Duty-Cycle & & $\gamma$ & Exponent (non-linear hydraulic & (---) \\
\hline$E E$ & Electro-explosive & $(--)$ & $\gamma$ & resistance) & \\
\hline$H$ & Height of the water column inside the & $(\mathrm{m})$ & $\eta$ & Operationalefficiency(temperature gain) & $(---)$ \\
\hline & barrel & (III) & $\theta$ & EED temperature increment (above & $\left({ }^{\circ} \mathrm{C}\right)$ \\
\hline$H_{b}$ & Height of the barrel & $(\mathrm{m})$ & & ambient temperature) & \\
\hline$i$ & Input & $(--)$ & $\pi$ & $\approx 3.1415$ & $(---)$ \\
\hline$n$ & Cycle counting (pulsed excitation) & $(---)$ & $\rho$ & Water density & $\left(\mathrm{kg} \cdot \mathrm{m}^{-3}\right)$ \\
\hline$o$ & Output & $(--)$ & $\tau_{b}$ & Barrel time constant & $(\mathrm{s})$ \\
\hline$P_{\text {ave }}$ & Average power & $(\mathrm{W})$ & $\tau_{E E}$ & EED time constant & (s) \\
\hline
\end{tabular}

$P_{i}(t) \quad$ EED electrical input power $\quad(\mathrm{W})$

$P_{P} \quad$ Peak power $\quad$ (W)

$P_{W} \quad$ Pulse width (s)

$Q_{i} \quad$ Input volumetric flow rate $\quad\left(\mathrm{m}^{3} \cdot \mathrm{s}^{-1}\right)$

$Q_{o} \quad$ Output volumetric flow rate $\quad\left(\mathrm{m}^{3} \cdot \mathrm{s}^{-1}\right)$

$r \quad$ Radius of the barrel (m)

$R \quad$ Hydraulic resistance $\quad\left(\mathrm{m}^{-2} . \mathrm{s}\right)$

$R_{T} \quad$ Thermal resistance $\quad\left({ }^{\circ} \mathrm{C}^{-1} \cdot \mathrm{W}\right)$

$V \quad$ Volume of water inside the barrel $\left(\mathrm{m}^{3}\right)$

\section{INTRODUCTION}

In order to study the ignition process of electro-explosive devices (EEDs), a consistent model is needed, one capable of coherently reproducing the temperatures of EDDs in response to the electrical power applied to them. In fact, EEDs merely convert energy from one type to another, which means that their electro-thermal model is a direct consequence of the energy conservation principle.

There is a complete theory behind such model and it is

Received: 04/11/11. Accepted: 23/01/12

*author for correspondence: carvalho@ita.br/Pç. Mal. Eduardo Gomes, 50. CEP: 12.228-900 - São José dos Campos/SP - Brazil founded on Rosenthal's (1961) and Prince \& Leeuw's (1988) work. Hoberman (1965) and Potter \& Scott (2004) developed 
a comprehensive study of this matter too. However, as the focus here is on the application of the model, not in the theory sustaining it, only the necessary level of abstraction will be retained from the original effort of those researchers and authors.

From the solution of that model, an outstanding advantage, in terms of temperature gain, will be noticed in favor of the electrical pulsed excitation (time to ignition not been a problem) whenever the width and period of the train of pulses are adjusted, taking into consideration the EED thermal time constant $\tau_{\mathrm{EE}}$. Thus the choice of the most appropriate power source (continuous or pulsed) to drive a specific EED will be primarily affected by its thermal time constant. Accordingly, the thorough knowledge of this constant will be essential for reliable and risk free (secure) applications of EEDs.

In this way, the steps to be followed to succeed in reaching the analysis of the EED ignition are: to adopt a simplified version of the currently used electro-thermal model of EEDs (an ordinary differential equation - an ODE, for short); to solve that model for different sorts of excitation (continuous and pulsed); and to verify the strong influence of both the EED (thermal time constant) and power source (pulse width and period of the train of pulses) parameters in the EED temperature response.

It follows that the key objective of the analysis to be carried out later in this paper is to guide users of EEDs, when faced with the choice of the right source of power to the right squib (vice-versa).

But, before analyzing the electro-explosive as an energy converter device, the investigation of a topic much more familiar to everyone, the barrel problem (Moran and Shapiro, 2008), can be valuable.

\section{A CONVENIENT ANALOGY}

Consider a barrel (a cylinder with transversal area, $\pi r^{2}$, height, $H_{b}$, and top surface removed), initially empty, having a small hole in its base. Assume a given steady water flow into the barrel. Naturally, a question will arise: how is the level of the water inside the barrel going to evolve as a function of time?

To facilitate the analysis of this problem, let the hole at the barrel base be modeled by a linear hydraulic resistance $R$ (actually, $R=R_{0} \cdot H^{(1-\gamma)}$, with $\gamma$ adjusted in accordance with each specific situation (Cochin, 1980), and let the water flux through the hole, $Q_{o}$, be proportional to $H$ :
$Q_{0}=\frac{H}{R}=\frac{H}{R_{0} \cdot H^{(1-\gamma)}}=\left.\frac{H^{\gamma}}{R_{0}}\right|_{\gamma=1}=\frac{H}{R_{0}}$

On the other hand, the water volumetric flow into the barrel is constant and equal to $Q$. Now, making use of the facts that, from the cylindrical geometry, $d V / d t=\pi r^{2} \cdot d H / d t$, and, from the mass-rate balance principle, $\rho \cdot d V / d t=\rho \cdot\left(Q_{i}-Q_{o}\right)$, the ODE describing the barrel problem is

$\frac{d H}{d t}+\frac{H}{\tau_{b}}=\frac{Q_{i}}{\pi r^{2}} \quad\left(\tau_{b}=R_{0} \cdot \pi r^{2}\right)$

This ODE has $H=Q_{i} \cdot R_{0} \cdot\left(1-e^{-\frac{t}{\tau b}}\right)$ for solution $(\mathrm{t}>0)$, from which the necessary condition for water to overflow is $Q_{i}>H_{b} / R_{0}$. But what barrels have to do with EEDs? Next section comes out to unveil this seeming mystery.

\section{THE ANALYSIS OF THE EED THERMAL MODEL}

By coating an electric resistance with a thick layer of high sensitivity explosive and applying an electric current to this circuit (bridgewire), the tiny explosive mass can certainly be initiated, but what is the best way to do that, by continuous or pulsed excitation? To answer this question, a simplified representation of the thermal phenomena involved in the EED ignition will be depicted.

Applying the energy-rate balance principle to the EED, the injected energy rate must be equal to the stored and the dissipated energy rates added together or, as in Eq. 3,

$P_{i}=C_{T} \cdot \frac{d \theta}{d t}+\frac{\theta}{R T} \Rightarrow \frac{d \theta}{d t}+\frac{\theta}{\tau_{E E}}=\frac{P_{i}}{C_{T}}$

$\left(\tau_{E E}=R_{T} \cdot C_{T}\right)$

The electro-thermal model corresponding to this differential equation is given in Fig. 1 (for experimental procedures to determine $\tau_{E E}=R_{t} \cdot C_{t}$, see Rosenthal's (1961) and Prince and Leeuw's (1988)).

Hence, the EED temperature behaves exactly as the level of the fluid accumulated inside the barrel, since differential Eq. 2 and Eq. 3, which represent both phenomena, hydraulic and thermal, are analogous. As a matter of fact, this similarity will be very useful in grasping the subtleties of the heat transfer process involved in the EED ignition.

As it has already been shown,

$\theta(t)=P_{i} \cdot R_{T} \cdot\left(1-e^{-\frac{t}{\tau_{E E}}}\right)$ 


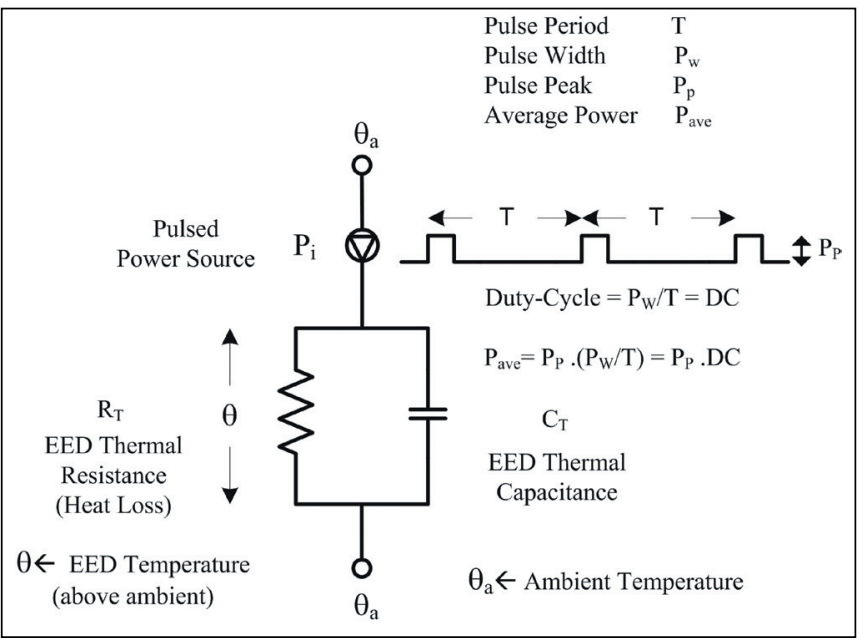

Figure 1. EED model.

Equation 4 is the solution of Eq.3, where $\mathrm{P}_{i}$ is constant. Therefore, the EED temperature is driven by the injected power.

From Eq.4, with $t \rightarrow \infty$, the maximum temperature (continuous input) is given by

$\theta_{\text {MAXCONT }}=P_{i} \cdot R_{T}=P_{\text {ave }} \cdot R_{T}$

Note how the EED temperature, due to the heat dissipation to the environment, via $R_{T}$, does not increase indefinitely - it is bounded (Fig. 2). Nevertheless, it suffices to make $\theta_{\text {MAXCONT }}>\theta_{\text {THRESHOLD }}$, by adjusting $P_{i}$, for ignition to occur.

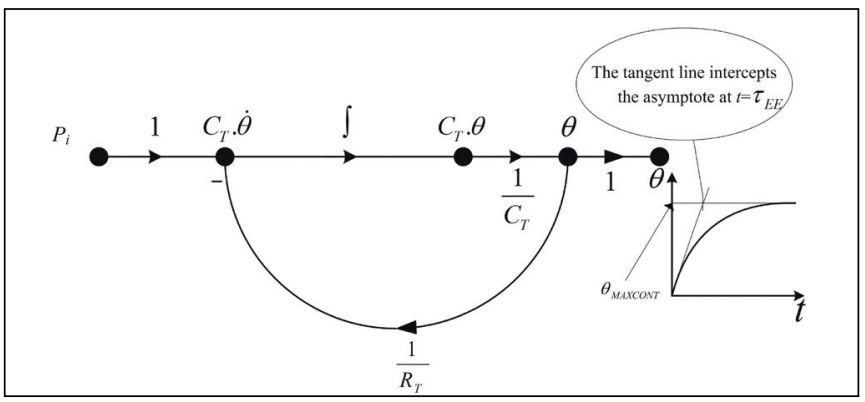

Figure 2. Signal flow graph (SFG) corresponding to Eq. 3 - the integrating effect of the thermal capacitance $\left(C_{T}\right)$ is counterbalanced by the heat conduction to the surroundings $\left(1 / R_{T}\right)$.

What happens when $P_{i}(t)$ is a train of power pulses (a switched power supply, for instance)? Despite the incontestable simplicity of the model adapted to our purposes, in this case, a certain mathematical expertise was required to solve it. For the complete solution of the EED ODE with a pulsed forcing function, the Laplace transform method should be used (Wylie, 1975). Here, one is going to derive an approximated solution in the time domain.

From the theory of differential equations (Wylie, 1975), the total temperature can be factored into transient (the transient part is always negative and steadily approaches zero) and periodic components. However, after a large number of pulses ( $n>>1, n$ is the pulse-cycle counting), the transient portion will have faded into insignificance (Fig. 3).

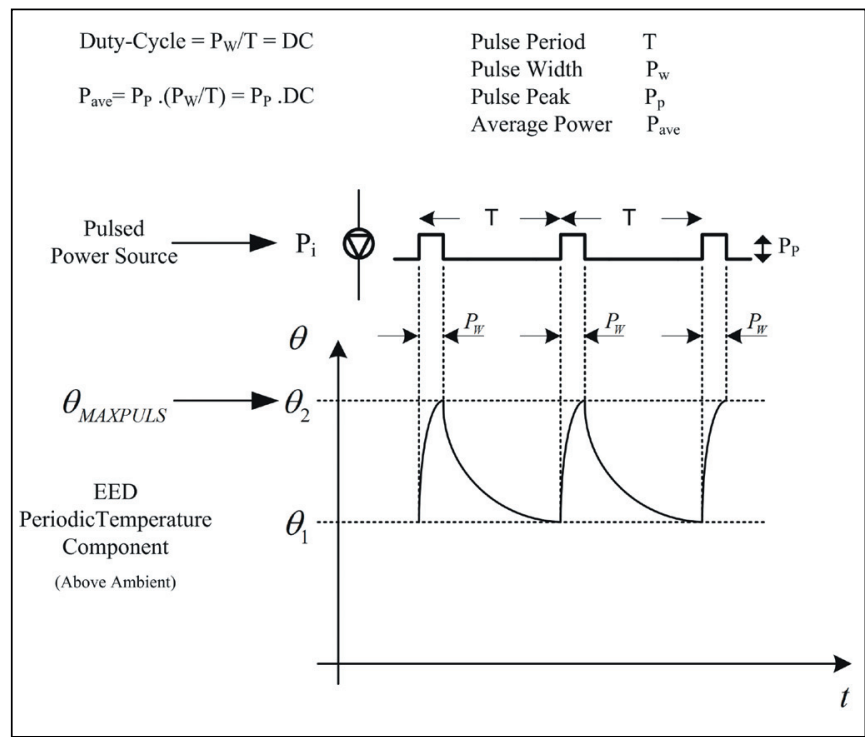

Figure 3. EED temperature after the settle down of the transient phase (pulsed excitation).

The problem was thus reduced to solve the following system of two ODEs with their associated boundary conditions (considering only the periodic component):

$\left\{\begin{array}{l}\frac{d \theta}{d t}+\frac{\theta}{\tau_{E E}}=\frac{P_{P}}{C_{T}},\left[0 \leq t \leq P_{W}\right] \wedge\left[\theta(0)=\theta_{1}, \theta\left(P_{W}\right)=\theta_{2}\right] \\ \frac{d \theta}{d t}+\frac{\theta}{\tau_{E E}}=0,\left[P_{W} \leq t \leq T\right] \wedge\left[\theta\left(P_{W}\right)=\theta_{2}, \theta(T)=\theta_{1}\right]\end{array}\right.$

From Eq. 6, it is not difficult to show that $\theta_{1}$ and $\theta_{2}$ must satisfy Eq. 7:

$\left\{\begin{array}{l}\theta_{2}-\left(e^{-\frac{P_{W}}{\tau E E}}\right) \cdot \theta_{1}=P_{P} \cdot R_{T} \cdot\left(1-e^{-\frac{P_{W}}{\tau E E}}\right) \\ \left(e^{\frac{\left(T-P_{w}\right)}{\tau E E}}\right) \cdot \theta_{2}-\theta_{1}=0 ;\end{array}\right.$

Solving Eq. 7 for $\theta_{2}=\theta_{\text {MAXPULS }}$ (maximum temperature):

$\theta_{M A X P U L S}=\theta_{2}=P_{P} \cdot R_{T} \cdot\left(\frac{1-e^{-\frac{P_{W}}{\tau_{E E}}}}{1-e^{-\frac{T}{\tau_{E E}}}}\right)$ 
Equation 8 , with $P_{\text {ave }}=P_{p} \cdot D C=P_{p} \cdot\left(P_{w} / T\right)$ fixed, becomes Eq. 9:

$\theta_{\text {MAXPULS }}=\theta_{\text {MAXCONT }} \cdot \frac{1}{D C} \cdot\left(\frac{1-e^{-\frac{T}{\tau E E} \cdot D C}}{1-e^{-\frac{T}{\tau E E}}}\right)$

Since (see Fig. 1 and Fig. 3)

$$
\begin{gathered}
P_{P}=P_{P} \cdot \frac{\frac{P_{W}}{\frac{P_{W}}{T}}=\frac{P_{P} \cdot \frac{P_{W}}{T}}{\frac{P_{W}}{T}}=\frac{P_{\text {ave }}}{\frac{P_{W}}{T}}=\frac{P_{\text {ave }}}{D C},}{\theta_{M A X C O N T}=P_{\text {ave }} \cdot R_{T},} \\
P_{P} \cdot R_{T}=\frac{P_{\text {ave }}}{D C} \cdot R_{T}=P_{\text {ave }} \cdot R_{T} \cdot \frac{1}{D C}=\theta_{M A X C O N T} \cdot \frac{1}{D C} \text {, and } \\
\frac{P_{W}}{\tau_{E E}}=\frac{T}{\tau_{E E}} \cdot \frac{P_{W}}{T}=\frac{T}{\tau_{E E}} \cdot D C .
\end{gathered}
$$

Now, defining the operational efficiency $\eta$ (a ratio of two temperatures, a kind of temperature gain) as in Eq. 10,

$\eta=\frac{\theta_{\text {MAXPULS }}}{\theta_{M A X C O N T}}=\frac{1}{D C} \cdot\left(\frac{1-e^{-\frac{T}{\tau E E} \cdot D C}}{1-e^{-\frac{T}{\tau E E}}}\right)$,

it is evident that, for certain combinations of $D C$ and $T / \tau_{E E}$, $\eta$ can be made $>>1$ (Fig. 4).

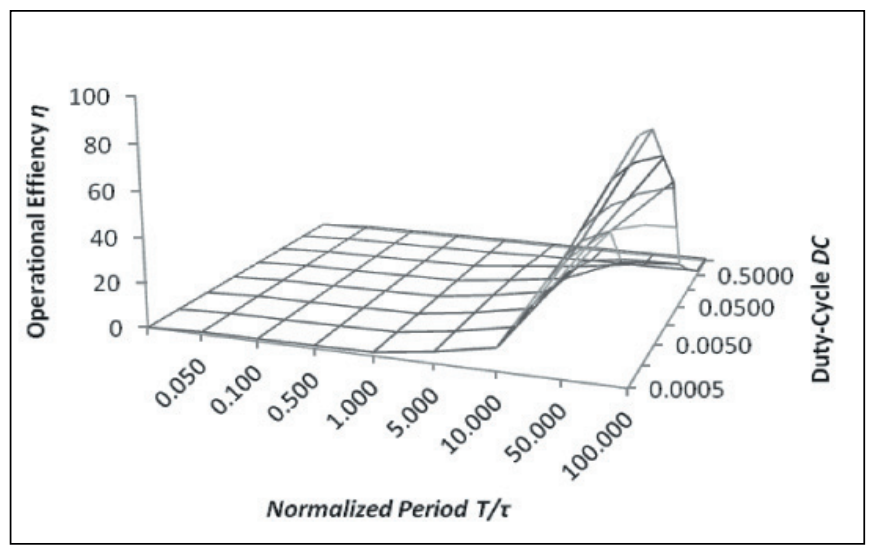

Figure 4. Operational efficiency $(\eta)$ as a function of the duty-cycle $(D C)$ and the normalized period $T / \tau_{E E}$.

Apparently, the pulsed excitation of the EED can be much more efficient in terms of the maximum attainable temperature (this is true only if $T$ is an independent variable, therefore under project control). In principle, for high efficiency, one just needs to operate the EED in the high $T / \tau_{E E}$ and low $D C=P_{w} / T$ domains (or short pulse domain). These conditions are equivalent to $\mathrm{T}>>\tau_{E E}>>$ $P_{w}$, since

$$
\left\{\begin{array}{l}
\left(\frac{T}{\tau_{E E}} \gg>1\right) \Rightarrow\left(T>>\tau_{E E}\right) \\
\left(\frac{P_{W}}{T}<<1\right) \Rightarrow\left(\frac{P_{W}}{\tau_{E E}} \cdot \frac{\tau_{E E}}{T}<<1\right) \Rightarrow\left(\frac{P_{W}}{\tau_{E E}}<<1\right) \Rightarrow\left(\tau_{E E}>>P_{W}\right)
\end{array}\right.
$$

Considering that both $P_{\text {ave }}=P_{p} \cdot D C=P_{p} \cdot\left(P_{w} / T\right)$ and $T$ are fixed, short pulses $\left(\mathrm{P}_{\mathrm{w}} \downarrow\right)$ also mean high peak-power $\left(\mathrm{P}_{\mathrm{P}} \uparrow\right)$. Therefore, this specific arrangement of $T / \tau_{E E}$ and $D C$ makes the train of pulses function as a train of impulses. Then, the EED is quasi-instantaneously responding to the energy of each incoming pulse. One can see that as follows: from Eq. 8, with $\mathrm{T}>>\tau_{E E}>>P_{w}$,

$$
\begin{aligned}
& \theta_{M A X P U L_{S}}=P_{P} \cdot R_{T} \cdot\left(\begin{array}{c}
\cong 1-\frac{P_{w}}{\tau_{E E}} \\
1-e^{-\frac{P_{w}}{\tau_{E E}}} \\
1-e^{-\frac{T}{\tau_{E E}}} \\
\cong 0
\end{array}\right) \cong P_{P} \cdot R_{T} \cdot\left[\frac{1-\left(1-\frac{P_{w}}{\tau_{E E}}\right)}{1}\right] \\
& \cong P_{P} \cdot R_{T} \cdot \frac{P_{w}}{\tau_{E E}} R_{T \cdot C T} \\
& \cong \frac{1}{C_{T}} \cdot \overbrace{P_{P} \cdot P_{\mathrm{W}}}^{\text {Pulse Energy }} \Rightarrow
\end{aligned}
$$

$\theta_{\text {MAXPULS }} \propto$ Pulse Energy

This is exactly the case of electrostatic discharges (ESD), actuating as $\mathrm{P}_{\mathrm{i}}(\mathrm{t})$, whenever $C_{T}$ is small and $R_{T}$ is large, a narrow pulse of high energy, an impulse for all practical purposes, can cause a rapid squib temperature increase, since the EED will not be able to dissipate, through $1 / R_{T}$, the energy delivered to it, and the feedback loop in Fig. 2 can be ignored (by rapidly discharging a huge amount of water into a barrel, the liquid reaches its top almost instantaneously, because the leak through the hole at its base is irrelevant).

Likewise, the operation of electrical capacitive discharge fuses, a particular case of the pulsed excitation (a pulsed excitation with $T \rightarrow \infty, P_{P} \rightarrow \infty$ and $P_{W} \rightarrow 0$ ), is now clear: almost all the thermal energy transferred to the EED will be converted, in just a single pulse, into a temperature increase, that is, 


$$
\begin{aligned}
& d \theta \cong \frac{P_{P}}{C_{T}} \stackrel{\int}{\longrightarrow} \\
& \int d \theta=\theta_{M A X P U L S E} \cong \theta_{\text {IMPULSE }} \cong \frac{1}{C T} \cdot \int_{0}^{P_{W}} P_{P} \mathrm{~d} t=\frac{1}{C T} \cdot P_{P} \cdot P_{W} \\
& \theta_{\text {MAXPULSE }} \cong \theta_{\text {IMPULSE }} \propto\left(P_{P} \cdot P_{W}\right) \Rightarrow \\
& \theta_{\text {MAXPULSE }} \cong \theta_{\text {IMPULSE }} \propto \text { Pulse Energy }
\end{aligned}
$$

Note that to calculate $\int_{\theta_{1}}^{\theta_{2}} d \theta=\theta_{2}-\theta_{1}=\theta_{\text {MAXPULS, the }}$ EED temperature $\theta_{1}$ (above ambient temperature), at the beginning of the power pulse,was assumed to be null (the EED was not able to stock-pile thermal energy during the previous cycles), a valid supposition, as $T>>\tau_{E E}$.

Once again, if $\theta_{\text {MAXPULSE }}>\theta_{\text {THRESHOLD }}$, ignition is assured (only one short high-energy pulse, one-shot trigger impluse, is sufficient to initiate the explosive charge).

Also, if $\tau_{E E}>>T>P_{W}$, then, from Eq. 6,

$\frac{d \theta}{d t}+\frac{\theta}{\tau_{E E}}=d \theta+\underbrace{\frac{\theta}{\tau_{E E}} d t}_{\text {negligible }}=\frac{P_{P}}{C_{T}} d t \Rightarrow$

$d \theta \cong \frac{P_{P}}{C_{T}} d t \stackrel{\int}{\longrightarrow} \Delta \theta \cong \frac{P_{P}}{C_{T}} \cdot \Delta t$

and the EED temperature increases progressively by, $\Delta \theta=\left(P_{P} / C_{T}\right) \cdot P_{W} \quad$ whenever an electric pulse of peak power $P_{P}$ (no further assumption on the peak power magnitude was necessary) and duration $P_{W}$ is applied to it (between pulses, $d \theta \cong 0$, and the EED temperature, $\theta$, remains approximately constant). After many pulses, therefore, relatively slowly, the temperature $\theta$ can certainly cross the ignition threshold, causing, without notice, the squib initiation. This adiabatic effect (or temperature stacking - the EED is storing thermal energy) is nothing else than a gradual accumulation of thermal energy, cycle after cycle. The EED has become, via $C_{T}$, a good longterm temperature integrator (a large, in diameter, barrel with a very small role in its base can gradually hold water inside it, since $\tau_{b}=R_{0} \cdot \pi r^{2}$ ). This is a very interesting, singular EED behavior, with many important applications in the aerospace industry, because high peak power is no longer required.

Finally, if $\tau_{E E}$ is very small $\left(\tau_{E E}<<P_{W}\right)$, the EED temperature practically follows the source of electrical power pulses $P_{i}(\mathrm{t})$, once

$$
\underset{\text { negligible }}{\frac{d \theta}{d t}}+\frac{\theta}{\tau_{E E}}=\frac{P_{i}(t)}{C_{T}} \Rightarrow \frac{\theta}{\tau_{E E}} \cong \frac{P_{i}(t)}{C_{T}} \Rightarrow \theta \cong R_{T} \cdot P_{i}(t)
$$

Now, forcing $R_{T} \cdot P_{P}>\theta_{\text {THRESHOLD }}$, which will require high peak power $P_{P}$, ignition is assured (this corresponds to a barrel of small cross-section with a large hole in its base - the liquid level follows the input pattern).

\section{CONCLUSIONS AND FURTHER RESEARCH}

Under strict conditions, pulsed excitation of EEDs can be advantageous, provided $\tau_{E E}$ is known:

If $\tau_{E E}>>>P_{W}$ (with $T$ being an independent variable, therefore under project control), remember that temperature gain $\eta$ can be even notable, but high peak power will be demanded (remenber that $P_{a v e}$ is constant).

On the other hand, if $\tau_{E E}>T$, EED temperature can softly reach the ignition threshold (either by friend or foe action), after several cycles, due to the gradual integration of the energy conveyed by each pulse, a kind of discrete electrothermal energy pumping - the temperature stacking effect. If time-to-ignition is not critical, any reasonable amount of peak power will do.

And, if $\tau_{E E}<<P_{W}$, the EED temperature simply follows the source of electrical power pulses $P_{i}(\mathrm{t})$.

It is also possible to initiate EEDs after one single highenergy impulse (one-shot fuse operation), particularly when $C_{T}$ is small, and $R_{T}$ is large.

Certainly, for optimized operation, the electrical driving source shall match, in terms of either energy or power, EEDs distinguishing features.

Furthermore, EEDs shall be shielded from their environment (coded ignition commands shall always be the rule): each EED must have its own local driving circuit module, only the coded commands shall come from the outside, preventing their soft ignition by foe intervention.

Following this conservative line, a matter that indeed deserves further investigation is the EED (one without any kind of protection) sensitivity to electromagnetic radiation (radar-like pulse-modulated radio frequency). Although closely linked with the subject developed in this article, it does not come within its scope (Thompson, 1973).

\section{REFERENCES}

Cochin, I., 1980, “Analysis and Design of Dynamic Systems", New York, Harper \& Row, Publishers, pp. 211. 
Haberman, C. M., 1965, "Engineering Systems Analysis", Ohio, C.E. Merrill Books, pp. 20-49.

Moran, M.J., Shapiro, H.N., 2008, "Fundamentals of. Engineering Thermodynamics", New York, John Wiley \& Sons, pp. 131-132.

Potter, M. C., Scott, E. P., 2004, “Thermal Sciences: An Introduction to Thermodynamics, Fluid Mechanics, and Heat Transfer", Kentucky, Brooks \& Cole, pp. 71-135.

Prince, W. C., Leeuw, M. W., 1988, "Analysis of the Functioning of the Bridge-wire Igniters Based on the Fitted
Wire Model", Propellants, Explosives, Pyrotechnics, Vol. 13, No. 4, pp. 120-125.

Rosenthal, L. A., 1961, "Thermal Response of Bridge-wires Used in Electro-explosive Devices”, Rev Sci Instrum, Vol. 32, No. 9, pp. 1033-1036.

Thompson, R. H., 1973, "Evaluation and Determination of Sensitivity and Electromagnetic Interactions of Commercial Blasting CAPS", USBM, Washington, D. C., Rep. no. F-C3102.

Wylie, C. R., 1975, “Advanced Engineering Mathematics”, Tokyo, McGraw-Hill Kogakusha, 4th ed., pp. 295-302. 